ÉGYPTE

monde arabe

\section{Égypte/Monde arabe}

15-16 | 1993

Les crises soudanaises des années 80

\title{
Les inondations de Khartoum en 1988 : impact et réaction des populations
}

\section{Mustafa Babiker}

\section{(2) OpenEdition}

\section{Journals}

Édition électronique

URL : https://journals.openedition.org/ema/1022

DOI : 10.4000/ema.1022

ISSN : 2090-7273

\section{Éditeur}

CEDEJ - Centre d'études et de documentation économiques juridiques et sociales

\section{Édition imprimée}

Date de publication : 31 décembre 1993

Pagination : 137-152

ISSN : 1110-5097

\section{Référence électronique}

Mustafa Babiker, "Les inondations de Khartoum en 1988 : impact et réaction des populations »,

Égypte/Monde arabe [En ligne], 15-16 | 1993, mis en ligne le 08 juillet 2008, consulté le 07 juillet 2022. URL : http://journals.openedition.org/ema/1022 ; DOI : https://doi.org/10.4000/ema.1022

Ce document a été généré automatiquement le 7 juillet 2022.

Tous droits réservés 


\title{
Les inondations de Khartoum en 1988 : impact et réaction des populations
}

\author{
Mustafa Babiker
}

\section{NOTE DE L'ÉDITEUR}

Traduit de l'anglais par François Ireton.

1 L'été 1988 fut à tous égards une redoutable saison de désastres au Soudan. Les pluies torrentielles et les terribles inondations qui frappèrent Khartoum furent sans précédent dans l'histoire plus que centenaire de la cité. Dans la nuit du 4 au 5 août, par exempte, la pluviométrie atteignit le record de $200 \mathrm{~mm}$. En trois semaines, au mois d'août, il tomba plus d'eau sur la capitale qu'il n'en était tombé durant les deux saisons des pluies précédentes. Plus de $80 \%$ de la surface de la ville furent submergés par les eaux, approximativement 200.000 logements et abris furent détruits et plusieurs zones d'habitation durent être totalement ou partiellement abandonnées par leurs habitants. L'invasion par les eaux de plusieurs centaines de fosses d'aisance déclencha sur toute la cité une très sérieuse menace sanitaire. Le caractère désastreux de ces événements se manifesta particulièrement dans l'effondrement total de services publics vitaux déjà très dégradés. Durant plusieurs jours, les systèmes de transports et de communication cessèrent complètement de fonctionner, eau et électricité furent coupés.

Dans les zones de la capitale directement touchées, les effets de la catastrophe ne tardèrent pas à se manifester, à différents degrés et sous différentes formes: augmentation de la mortalité, déclenchement d'épidémies, destruction, sous-nutrition et multiplication des sans-abri. Bien évidemment, la majorité des victimes appartenaient aux classes les plus pauvres et en particulier aux groupes de personnes déplacées du sud, habitant les camps de réfugiés de la périphérie de Khartoum. Dans la plupart de ces camps, la population se retrouva sans logement, sans eau potable et sans 
secours alimentaires suffisants. Les mêmes zones furent directement menacées par de très sérieux risques sanitaires, et de nombreux cas de dysenterie furent enregistrés ; dans de telles conditions, l'on pouvait craindre l'apparition du choléra.

3 Aux inévitables questions portant sur la responsabilité des conséquences - d'une ampleur inouïe - des inondations, maintes accusations surgirent, qui émanaient aussi bien d'hommes politiques, de membres de l'administration publique et d'organisations gouvernementales que de journalistes et d'universitaires, tous pointant un doigt vengeur dans toutes les directions... à l'exception de la leur.

Dans cette étude, on tentera de résister, autant que taire se peut, à la tentation de débusquer des coupables. On essaiera plutôt de tirer les leçons d'un tel désastre, avec la ferme conviction que les sciences sociales peuvent contribuer à résoudre les problèmes liés aux crises à fondement écologique, ne serait-ce qu'en montrant la complexité des relations existant entre des événements "naturels » donnés et les types de réponses qu'y apportent des collectivités locales particulières. En l'occurrence, il s'agira ici d'analyser comment l'une d'elles a réagi, en l'absence - ou en la présence peu «efficace " - d'une intervention extérieure, à une "pression écologique » qui a, semble-t-il, poussé la population au-delà des limites de ses «capacités adaptatives ». L'on prendra pour cela l'exemple d'un quartier informel situé dans la périphérie sud de Khartoum, Deim Mayo'.

5 Il n'est pas question ici de considérer Deim Mayo comme une entité monolithique dont les habitants auraient réagi de façon uniforme aux conséquences des inondations de 1988. L'on a plutôt tenté d'analyser les variations de mode et de capacité de réponse des habitants de ce quartier face au désastre frappant leur nouvel environnement urbain, cela en termes d'origine ethnique et régionale et en fonction de l'histoire de leur installation à Khartoum. Pour cela, on a d'abord pris en compte la bipartition de Deim Mayo : d'un côté, l'ancienne implantation (le Vieux-Mayo); de l'autre côté, le plus récent (Al-Sahra'), majoritairement peuplé de personnes déplacées venues du sud du Soudan. Ceci ne signifie pas que l'ensemble géographique enquêté soit habité par deux catégories socio-ethniques exclusives. Cette première distinction vise seulement à permettre d'identifier les sous-groupes sociaux les plus vulnérables et à explorer les modes de réponse différenciés à la catastrophe.

6 Cette étude se compose de trois parties. L'on donne d'abord des informations de base sur les principaux aspects démographiques et socio-économiques de la zone d'enquête," puis l'on décrit les principales conséquences des inondations sur les conditions d'existence de ses habitants. Dans un troisième temps, l'on examine de façon détaillée les réponses de la population elle-même et les interventions externes, ainsi que les évaluations que les habitants font de ces dernières quant à leur délai, leur ampleur et leur efficacité.

Données démographiques et socio-économiques sur la localité de Deim Mayo

7 La localité de Deim Mayo est située à quatre kilomètres environs du centre de Khartoum, dans les franges sud de la cité. Selon le recensement national de 1983, la population totale de cette zone était de 36.000 habitants, répartie dans 8.600 logements. Les estimations démographiques pour 1988 donnaient un effectif de 46.400 habitants, largement constitué de migrants arrivés à Khartoum vers la fin des années 60 , en provenance des provinces de l'ouest à la suite de la sécheresse, et des provinces du sud où sévissait la guerre civile. Ces déplacés ont d'abord vécu dans d'autres bidonvilles situés en différents points de la capitale (dont beaucoup en bordure de la zone 
industrielle de Khartoum) et ont été ensuite transférés par l'État à Deim Mayo au début des années 70.

8 En menant cette opération de relogement, l'administration poursuivait deux buts: d'une part, libérer des zones qui étaient considérées par l'Etal comme propices à l'implantation d'établissements industriels et à l'extension planifiée de l'habitat; d'autre part, regrouper les zones d'urbanisation informelle afin de doter leurs anciens occupants des équipements et services publics indispensables. Le premier objectif atteint, l'État eut moins de succès dans la réalisation du second, qui constituait pourtant l'argument principal pour justifier le déplacement forcé de ces occupants. Ainsi, de récents rapports sur les conditions socio-économiques d'existence de la population de Deim Mayo soulignaient la quasi-absence de services sociaux dans ce quartier. De plus, le fait que ce dernier, ait été l'une des zones d'urbanisation les plus touchées par les conséquences de l'inondation de 1988 confirme bien l'état lamentable des quelques équipements et services qui y avaient été implantés. Pourtant, durant les années 1984-1987, le nombre d'habitants de Deim Mayo avait nettement augmenté, de nombreux réfugiés du Sud - suite à la reprise de la guerre civile - et des victimes de la sécheresse de l'ouest qui avait sévit en 1984-85 ayant rejoint la population déjà implantée sur les lieux.

9 Les premiers arrivants de cette "seconde vague " s'installèrent dans la partie déjà habitée de Deim Mayo, et les immigrés plus tardifs étendirent le quartier au sud de la zone déjà urbanisée. Cette extension, presque entièrement peuplée de réfugiés du sud, fut baptisée 'Al-Sahra' », qui signifie littéralement « zone désertique ».

qui concerne ses origines régionales, la population de Deim Mayo est donc très majoritairement issue de l'ouest (régions du Kordofan et du Darfour) et du sud: les natifs de ces deux régions forment $79 \%$ de la population de ce quartier. Les habitants originaires du nord, de l'est et du centre n'en constituent que $9 \%$ et le reste provient d'un certain nombre de pays voisins du Soudan - Tchad, Zaïre et même Nigeria habitant tous le "Vieux Mayo». Seule une infime partie des résidants est née à Khartoum (hormis les enfants des habitants originaires des autres parties du pays, qui ont été comptés ici avec leurs parents).

Lors d'une enquête récente, 42 ethnies d'appartenance ont été recensées à Deim Mayo (cf. Rapport, 1989) dont les plus représentées sont, pour celles du sud, les Dinka, Nuer, Shilluk, Zandé, Toposa, Baria et Fertit et, pour celles de l'ouest, les Nouba, Four, Masalit, Zaghawa et Dajo (ethnies non arabes) et les Ta'isha, Bedairiya, Hamar, Gawama'a, Béni Halba, Rezeigat, Manasra et Meseiriya («tribus» arabes). Les principales ethnies d'Afrique de l'Ouest représentées sont les Hausa, les Guraan et les Bemo. Chacune des 22 communautés de voisinage de Deim Mayo a à sa tête un chaykh ou un chef, désigné d'après le groupe ethnique dominant de la communauté de voisinage dont il est responsable; on parle ainsi du chef des Nouba, des Hausa, des Junubiyyin (originaires du Sud-Soudan), etc.

Impact sur Deim Mayo des inondations de 1988

12 Comme les autres zones d'habitat "spontané » des franges périphériques de Khartoum, établies sur des terres de basse altitude, Deim Mayo fut très sérieusement affectée par les pluies torrentielles et les inondations qui s'ensuivirent. Des centaines de logements y furent détruits, les familles perdant non seulement leur abri mais encore tout ce qui leur appartenait. Le tableau 1 (page suivante) résume le type de pertes subies dans un 
échantillon de 492 personnes interrogées, parmi lesquelles 401 habitaient le VieuxMayo et les autres Al-Sahra'.

Etant donné que le type de logement dominant à Deim Mayo est constitué de maisons en terre, et particulièrement de briques de boue séchées au soleil, il n'est pas surprenant que $14 \%$ de ces logements aient été totalement détruits par l'inondation et que $58 \%$ des autres l'aient été partiellement. La proportion de destruction, totale ( $28 \%$ des logements) ou partielle, est encore plus forte à Al-Sahra', où s'étaient récemment installés les déplacés venus du sud, la plupart des abris construits par eux étant faits de cartons et de divers matériaux de récupération qui ne résistèrent pas aux pluies diluviennes.

Tableau 1 : Répartition des personnes enquêtées selon la gravité des pertes subies (\%)

\begin{tabular}{|l||l|l|l|}
\hline Types de dommages & $\mathrm{N}=402$ & $\mathrm{~N}=90$ & $\mathrm{~N}=492$ \\
\cline { 2 - 4 } & Quartier ancien \% & Al-Sahra \% & Deim Mayo \% \\
\hline \hline Maison détruite & 10.9 & 27,8 & 14.0 \\
\hline \hline Maison endommagée & 56.9 & 61.2 & 57.7 \\
\hline \hline Autres dommages & 18.5 & 5.5 & 116.1 \\
\hline \hline Sans objet & 13.7 & 5.5 & 12.2 \\
\hline \hline Total & 100.0 & 100.0 & 100.0 \\
\hline
\end{tabular}

Source : Babiker \& Kamir $1989: 82$.

14 Hormis la perte du logement, conséquence la plus grave des inondations, les personnes enquêtées évoquent fréquemment les difficultés d'accès à l'eau potable et à l'alimentation. S'agissant de l'eau potable, étant donné qu'il n'y avait que deux puits forés à Deim Mayo, la majorité des habitants avait recours aux transporteurs d'eau privés. A la suite des inondations, les prix pratiqués par ces derniers grimpèrent vertigineusement, certains ménages payant jusqu'à l'équivalent de la moitié de leur revenu journalier pour acquérir de quoi satisfaire leurs faibles besoins en eau. La population déplacée, récemment arrivée à Deim Mayo, est bien sûr la première à avoir fait les frais d'une telle hausse. Du reste, les prix étaient si élevés et les quantités d'eau disponibles si limitées que le consommation fut réduite au strict minimum : boisson et cuisson des aliments.

A Deim Mayo, l'approvisionnement alimentaire demeura un problème majeur durant les inondations et au-delà, moins du fait de pénuries dans ce domaine qu'en raison du niveau des prix. Les opérations menées par les ONG pour assurer le ravitaillement de la population n'eurent qu'une influence marginale sur les prix. Les conséquences nutritionnelles de cette détérioration de la situation alimentaire sont illustrées par le fait que l'on enregistra des taux allant de 15 à $33 \%$ d'enfants de moins de cinq ans 
présentant un indice de sous-nutrition important (rapport poids/taille inférieur à $80 \%$ de la normale).

Une autre conséquence nocive des inondations réside dans les problèmes sanitaires que rencontra la population. La malaria, par exemple, affecta de quatre à cinq fois plus d'habitants qu'elle ne le faisait en temps normal, les eaux stagnantes constituant un milieu tout à fait propice au développement des larves de moustiques. Les travailleurs $\mathrm{du}$ domaine sanitaire rapportèrent que beaucoup d'autres maladies atteignirent un taux d'incidence très supérieur à la moyenne, en particulier la dysenterie, la typhoïde, différentes formes d'anémie et des infections des voies respiratoires supérieures. Le tableau 2 présente la répartition des familles selon les maladies qui ont été contractées en leur sein à la suite des inondations.

Tableau 2 : Répartition des familles par type de maladie contractée durant les inondations (\%)

\begin{tabular}{|c|c|c|c|}
\hline \multirow[t]{2}{*}{ Maladies } & $\mathrm{N}=402$ & $\mathrm{~N}=90$ & $\mathrm{~N}=492$ \\
\hline & Ancien quartier \% & Al-Sahra \% & Deim Mayo \% \\
\hline Malaria & 43.6 & 50.1 & 44.8 \\
\hline Anémie & 0.2 & 1.1 & 0.4 \\
\hline Typhoïde & 0.7 & 3.3 & 1.2 \\
\hline Dysenterie & 1.0 & 5.5 & 1.8 \\
\hline Rougeole & 1.2 & 1.1 & 1.2 \\
\hline Dermatoses & 2.6 & 7.8 & 3.4 \\
\hline Blessures & 1.2 & 1.1 & 1.2 \\
\hline Plusieurs & 19.7 & 7.7 & 17.8 \\
\hline Autres & 15.2 & 11.1 & 14.4 \\
\hline Sans objet & 14.4 & 11.2 & 13.8 \\
\hline Total & 100.0 & 100.0 & 100.0 \\
\hline
\end{tabular}

Source : Babiker \& Kamir 1989 : 85.

Le tableau indique clairement qu'à Deim Mayo, 86 \% des personnes enquêtées ou des membres de leur famille ont contracté au moins une maladie durant la catastrophe de 1988. Ce tableau confirme par ailleurs les affirmations des travailleurs de la santé selon lesquelles la malaria, présente à ce moment-là dans $45 \%$ des familles, fut la maladie la plus répandue. 
18 L'impact de longue durée des inondations reste encore à mesurer. Les rapports des ONG mettent l'accent sur la persistance d'un haut niveau d'incidence des maladies intestinales et sur la remontée des taux de malnutrition. Alors qu'une part des cas de dysenterie s'explique par la dégradation de l'état nutritionnel, le facteur le plus important dans ce domaine réside dans les difficultés persistantes d'accès à une alimentation satisfaisante et à une eau vraiment potable.

Les réactions à la catastrophe

Dans les efforts déployés pour faire face aux problèmes posés par l'inondation, l'on peut distinguer deux aspects: les mécanismes d'auto-assistance et l'assistance extérieure.

Les mécanismes d'auto-assistance locale

20 Ces derniers comprennent des mécanismes de réponse au niveau des groupes domestiques individuels et des communautés de voisinage. Au premier de ces niveaux correspondent des réponses immédiates diverses dont le tableau 3 donne les types principaux.

Tableau 3 : Répartition des familles selon le type de réponse immédiate développée face à l'inondation et à ses conséquences (\%)

\begin{tabular}{|l|l|l|l|}
\hline Interventions & $\mathrm{N}=402$ & $\mathrm{~N}=90$ & $\mathrm{~N}=492$ \\
\hline \hline & $\begin{array}{l}\text { Ancien } \\
\text { quartier \% }\end{array}$ & $\begin{array}{l}\text { Al-Sahra' } \\
\%\end{array}$ & $\begin{array}{l}\text { Deim Mayo } \\
\%\end{array}$ \\
\hline $\begin{array}{l}\text { Pas d'intervention : auto-financement de la } \\
\text { reconstruction }\end{array}$ & 51.7 & 45.6 & 50.6 \\
\hline \hline Pas d'intervention, attente d'assistance extérieure & 18.2 & 16.9 \\
\hline \hline Reconstruction immédiate & 10.2 & 11.1 \\
\hline \hline Autres & 1.2 & 1.0 \\
\hline \hline Sans objet & 100.0 & 100.0 \\
\hline \hline Total & 20.0 & 100.0 \\
\hline
\end{tabular}

Source : Babiker \&. Kamir $1989: 85$.

21 Ce tableau indique que très peu de victimes des inondations abandonnèrent leur logement et qu'une proportion non négligeable de familles (12,4\%) s'employèrent aussitôt à se procurer les ressources nécessaires au démarrage de la reconstruction. Mais le retard apporté au démarrage de cette opération (67,5\% des cas) est un bon indicateur du fait que les pauvres étaient déjà marginalisés auparavant et qu'il leur était impossible de se mettre à reconstruire sans une assistance extérieure significative. Néanmoins, l'attitude majoritaire de ceux qui n'entreprirent pas immédiatement cette reconstruction fut une affirmation d'indépendance, les $50,6 \%$ des familles qui comptaient autofinancer cette opération excédant nettement les $17 \%$ qui décidèrent 
d'attendre pour ce faire une aide extérieure. Cette attitude majoritaire constitue la base sur laquelle s'appuyèrent les mécanismes d'auto-assistance familiale et communautaire.

Au niveau familial, ces mécanismes consistèrent en diverses activités, depuis les opérations de terrassement et d'endiguement, l'évacuation de l'eau ou la construction d'abris provisoires, jusqu'à la reconstruction des maisons et l'application de traitements médicaux populaires. Le tableau 4 donne la distribution des ménages selon les différents travaux entrepris par les familles elles-mêmes.

Tableau 4 : Répartition des ménages selon leurs interventions face aux conséquences des inondations

\begin{tabular}{|c|c|c|c|}
\hline \multirow[t]{2}{*}{ Interventions } & $\mathrm{N}=402$ & $\mathrm{~N}=90$ & $\mathrm{~N}=492$ \\
\hline & Ancien quartier \% & Al-Sahra \% & Deim Mayo \% \\
\hline Constr. de digues & 8.0 & 5.5 & 7.5 \\
\hline Evacuation de l'eau & 8.0 & 11.1 & 8.5 \\
\hline Abris temporaires & 8.9 & 17.9 & 10.6 \\
\hline Reconstruction & 19.9 & 28.9 & 21.5 \\
\hline Rien & 34.3 & 25.5 & 32.5 \\
\hline Abandon de maison & 1.0 & 4.4 & 1.7 \\
\hline Soins aux malades & 2.2 & 0.0 & 1.8 \\
\hline Digues + drainage & 12.7 & 5.5 & 12.3 \\
\hline Sans réponse & 4.0 & 2.2 & 3.6 \\
\hline Total & 100.0 & 100. & 100.0 \\
\hline
\end{tabular}

Source : Babiker \& Kamir 1989 : 89,

Les réponses relevant de l'auto-assistance des communautés de voisinage ont été l'œuvre de regroupements tels que les comités de chefs de communauté (et leurs assistants), les comités de jeunes, les associations de quartier, les comités pour le bien public et les associations à base ethnique. De nombreuses associations et comités ont été très actifs dans les opérations de premiers secours et d'évacuation des eaux, dans la distribution de nourriture et l'enregistrement des noms des victimes. A titre d'illustration, l'on donnera une brève description des actions menées par l'un de ces comités, le Conseil des représentants des tribus du Sud (CRTS).

L'idée de fonder ce comité se fit jour lorsque le nombre de personnes déplacées venues du sud s'accrut notablement à Deim Mayo. La diversité des ethnies représentées et le 
mode de distribution géographique des résidants du quartier appartenant à ces dernières créèrent diverses difficultés, en particulier en ce qui concerne la résolution des conflits de voisinage, qui devenaient vite des conflits interethniques. Le CRTS fut créé pour faire face à ces difficultés et chaque ethnie du sud fut représentée en son sein par le président de son Comité pour le bien public.

En mai 1988 et suite aux problèmes qu'avaient déjà posés les pluies de l'été 1987, un Comité des jeunes avait été fondé. Dès qu'il fut constaté que les pluies étaient torrentielles, le Comité des jeunes émit l'idée d'acquérir au plus vite l'équipement nécessaire à l'évacuation des eaux par les habitants eux-mêmes. Lé président du CRTS approuva l'initiative et convoqua une réunion de son Comité, qui accepta le projet et décida que chaque chef de ménage originaire du sud verserait 10 livres soudanaises de contribution à sa réalisation. Un montant de 1.312 livres fut ainsi recueilli et attribué au Comité des jeunes pour qu'il achète le matériel adéquat.

Lors des pluies de 1988 (la nuit du 4 au 5 août en particulier), le CRTS et le Comité des jeunes travaillèrent ensemble " au sifflet » et appelèrent la population à construire des digues permettant de canaliser les eaux qui déferlaient dans le quartier. Cette nuit-là, pas une seule maison ne s'effondra sur ses habitants, car ces derniers étaient tous dehors en train de travailler. Ceux qui perdirent leur abri (souvent des familles déplacées récemment) furent regroupés et logés provisoirement dans l'église. Les autorités ecclésiastiques leur firent attribuer tout le grain contenu dans leurs réserves (160 sacs de sorgho) qui fut distribué par le CRTS aux victimes, les représentants de chaque ethnie du sud le transmettant, avec l'aide des membres du Comité des jeunes, à leurs contribules respectifs. Une liste complète contenant les quantités de grains et les noms de ceux qui reçurent ces secours fut soumise aux autorités ecclésiastiques. De même, le président du CRTS, avec l'assistance du Comité des jeunes, prépara, à la requête de diverses $\mathrm{ONG}$, une liste des victimes qui servit ultérieurement à la distribution des secours.

Le scénario décrit plus haut n'est qu'un exemple d'initiative locale d'auto-assistance, dont l'objectif était de faire lace à une catastrophe " naturelle ». Le terme " naturelle » est mis ici entre guillemets pour la simple raison que, bien que tous les habitants de Khartoum aient subi les pluies torrentielles et les inondations d'août 1988, leur impact sur les différentes fractions de la population fut loin d'être uniforme. Cette caractéristique ne peut être expliquée qu'en référence à l'économie politique de la cité. Néanmoins, l'exemple du CRTS est instructif et apporte un intéressant contretémoignage vis-à-vis des descriptions que la littérature disponible donne des déplacés, invariablement présentés comme des victimes pliant l'échine sous le poids des forces « naturelles » et « historiques ».

L'assistance externe

Ce serait une grave erreur de présenter de façon romantique les mécanismes locaux d'auto-assistance. Ces derniers, malgré leurs mérites, ont bien évidemment leurs propres limites. Nulle part celles-ci ne sont aussi patentes que dans le domaine du relogement provisoire, des secours alimentaires et de la protection sanitaire. Au vu deces limitations, ce n'est pas une coïncidence si l'administration gouvernementale et les ONG intervinrent dans les zones sinistrées comme Deim Mayo pour y distribuer divers secours (nourriture, tentes, bâches de plastique, médicaments, etc.).

29 Cependant, à part un appel à l'aide internationale, la réponse officielle à la catastrophe - en termes de mobilisation des ressources comme d'intervention de personnel 
entraîné pour agir en de pareilles circonstances - peut être considérée comme ayant été pratiquement nulle dans beaucoup de quartiers. De plus, des critiques variées s'élevèrent contre la gestion des opérations de secours, spécialement lorsque ces dernières furent menées par les personnels des administrations locales, par les militaires et par des représentants des partis politiques. Ces critiques visent aussi bien l'inefficacité des systèmes de distribution des secours, la corruption ou le détournement de ces derniers, qui n'atteignirent pas les véritables victimes, que le favoritisme basé sur les origines ethniques, l'appartenance religieuse ou les affiliations politiques.

En fait, les agences fournisseuses d'aide, les ONG et les organisations internationales passèrent la seconde partie de l'année 1988 à accomplir diverses activités concernant la mobilisation de fonds, d'équipements, de moyens de transports et de personnel de secours afin d'intervenir le plus efficacement possible, tout en tentant de faire au plus vile. Des pays intervinrent à titre personnel, bien qu'il aille sans dire que, vu l'échelle du désastre, les fonds requis étaient considérables et que les réparations prendraient de nombreuses années. Néanmoins, des programmes médicaux à court terme (trois mois) furent organisés, incluant un personnel médical qualifié, des équipements et des moyens de distribution d'eau potable, ce qui relevait de la plus extrême urgence, vu le vecteur épidémique que constituait l'eau polluée utilisée par les sinistrés.

Ainsi, aussi justifiées fussent les critiques visant l'assistance extérieure, il est clair que sans les différentes formes qu'elle a prise, les conséquences de l'inondation - y compris les plus graves qui furent évitées, comme les épidémies mortelles - auraient été plus dramatiques encore.

Appréciations sur l'efficacité des interventions

On examinera ici les jugements que portèrent les habitants sur les mérites comparés des différents types d'aides dont ils furent les bénéficiaires. Il ne s'agit pas de classer «objectivement» les interventions quant à la qualité des prestations fournies, ni de décerner des prix aux "meilleures opérations de secours", mais de tirer des leçons pour l'avenir, à partir de ce que la population a perçu quant à leur efficacité et quant à leur complémentarité. L'observation concrète du déroulement des actions menées à Deim Mayo en est l'occasion.

Tableau 5 : Répartition des bénéficiaires de secours selon la source de l'assistance

\begin{tabular}{|c|c|c|c|}
\hline \multirow[t]{2}{*}{ Sources } & $\mathrm{N}=402$ & $\mathrm{~N}=90$ & $\mathrm{~N}=492$ \\
\hline & Ancien quartier \% & Al-Sahra \% & Deim Mayo \% \\
\hline ONG & 57.1 & 71.1 & 59.5 \\
\hline Pouvoirs publics & 8.7 & 0.0 & 7.2 \\
\hline Parenté & 3.7 & 4.4 & 3.8 \\
\hline Voisinage & 2.7 & 7.8 & 3.6 \\
\hline
\end{tabular}




\begin{tabular}{|l|l|l|l|}
\hline Sans objet & 27.8 & 16.7 & 25.9 \\
\hline \hline Total & 100.0 & 100.0 & 100.0 \\
\hline
\end{tabular}

Source : Babiker \& Kamir 1989: 95

Nous avons vu comment l'assistance apportée aux individus et aux familles est d'abord venue de la population du quartier elle-même, puis de l'extérieur (dans ce cas, de l'État soudanais pour une faible part et, pour une part notable, d'ONG et d'autres organismes, soudanais, étrangers ou internationaux). Le tableau 5 ci-dessus présente la distribution des familles bénéficiaires de secours selon ce qu'elles estiment être la source d'aide la plus importante parmi celles qui leur ont été apportées. Le tableau 5 montre que les ONG ont constitué indiscutablement la source d'aide essentielle, que se soit dans le Vieux-Mayo ou à Al-Sahra'. Les secours en provenance de l'État se sont limités au Vieux-Mayo. Quant à l'assistance apportée par des parents ou des voisins, bien qu'elle ne constitue qu'une faible part du total des aides, elle est relativement plus importante dans la seconde subdivision du quartier que dans la première.

Si nous considérons maintenant le critère des délais d'intervention et d'assistance aux victimes, il est normal que les secours en provenance du quartier lui-même - ce que nous avons appelé l'auto-assistance - aient été les plus prompts. Mais l'on constate une forte disparité de rythme d'intervention entre les divers organismes extérieurs au quartier: les ONG ont été les premières à arriver sur le terrain; quant aux interventions étatiques soudanaises, limitées ou inexistantes (comme ce fut le cas à AlSahra') elles ont atteint très tardivement les victimes (Rapport, 1989).

Nous avons jusqu'ici envisagé de manière globale les différentes formes de secours. $\mathrm{Si}$ l'on prend maintenant en exemple le problème du logement provisoire durant l'inondation, on s'aperçoit (tableau 6) que ce sont de nouveau les ONG qui ont fourni l'assistance la plus importante, particulièrement dans les zones d'urbanisation les plus récentes et en général les plus précaires. Ceci est tout à fait confirmé par le contenu de nos interviews non directifs, qui montrent que les tentes et les bâches en plastique offertes par les ONG sont parvenues dès les premiers jours aux sinistrés et ont constitué leurs seuls abris provisoires durant la période de reconstruction de leur logement.

Tableau 6 : Répartition des ménages en fonction de la source d'assistance en matière d'hébergement

\begin{tabular}{|l|l|l|l|}
\hline Source d'assistance & Ancien quartier \% & Al-Sahra \% & Deim Mayo \% \\
\hline \hline Pouvoirs publics & 4.3 & 0.0 & 3.5 \\
\hline \hline Voisinage & 16.9 & 11.1 & 15.8 \\
\hline \hline ONG & 15.9 & 32.3 & 18.7 \\
\hline \hline Sans objet & 59.9 & 52.2 & 58.6 \\
\hline \hline Sans réponse & 2.8 & 4.4 & 3.0 \\
\hline
\end{tabular}




\begin{tabular}{|l|l|l|l|}
\hline Total & 100.0 & 100.0 & 100.0 \\
\hline
\end{tabular}

Source : Babiker \& Kamir 1989:100.

Etant donné l'opinion tout à fait positive des enquêtes sur la rapidité et l'étendue des interventions des ONG (en matière de relogement provisoire en particulier), il est dans un premier temps curieux de constater que, malgré tout, s'agissant de l'efficacité des interventions, ce sont les actions basées sur les mécanismes d'auto-assistance qui l'emportent, tout particulièrement à Al-Sahra' où les habitants paraissent sceptiques quant à l'efficacité de toute aide extérieure. Ce qui n'est, à y réfléchir, pas tellement étonnant si l'on prend en compte le mode d'intervention spontané et immédiat presque préventif (construction de digues pendant les pluies torrentielles elles-mêmes) - que permettent ces mécanismes. La seule réserve manifestée par les enquêtes à l'égard des ONG vient du fait que celles-ci interviennent nécessairement plus tardivement et de manière plus sélective, en ce qui concerne aussi bien les domaines d'action que les familles concernées. De plus, les rations de sorgho, de lait déshydraté et d'huile alimentaire qu'ont distribuées les ONG l'ont souvent été en quantité insuffisante, du fait des lourdes contraintes et complications de tous ordres pesant sur la " chaîne logistique » d'approvisionnement. Il en a été en partie de même en ce qui concerne la distribution des tentes et des bâches en plastique ; le caractère parfois un peu chaotique de cette dernière a exaspéré ceux des habitants qui avaient perdu tout abri et qui continuaient à subir les pluies. Ces derniers étant souvent ceux dont les mécanismes d'auto-assistance se révélaient les moins capables de faire face à la détresse, il est logique qu'attendant davantage de la part des secours extérieurs, ils se soient montrés plus critiques à l'égard de leur organisation, ce que montre le tableau 7.

Tableau 7 : Distribution des opinions concernant les types de secours les moins efficaces

\begin{tabular}{|l||l|l|l|}
\hline Réponse & $\mathrm{N}=402$ & $\mathrm{~N}=90$ & $\mathrm{~N}=492$ \\
\cline { 2 - 4 } & Ancien quartier \% & Al-Sahra, \% & Deim Mayo \% \\
\hline \hline Premiers secours & 46.8 & 24.5. & 42.7 \\
\hline \hline Aide alimentaire & 28.4 & 33.3 & 29.3 \\
\hline \hline Aide sanitaire & 6.2 & 8.9 & 6,7 \\
\hline \hline Hébergement & 4.2 & 15.6 & 6.3 \\
\hline \hline Habillement & 4.7 & 3.2 & 4.5 \\
\hline \hline Aide en numéraire & 3.0 & 6.0 & 2.8 \\
\hline \hline Sans réponse & 6.7 & 12.2 & 7.7 \\
\hline
\end{tabular}




\begin{tabular}{|l|l|l|l|}
\hline Total & 100.0 & 100.0 & 100.0 \\
\hline
\end{tabular}

Source : Babiker \& Kamir $1989: 100$. non seulement fait depuis longtemps l'expérience de conditions de vie déplorables, mais encore étaient les moins à même de faire face à la catastrophe qu'engendrèrent les pluies torrentielles de 1986. Cependant, on l'a vu, en dépit de fortes limites techniques et de la faiblesse de leurs ressources, les habitants sont loin d'être restés passifs. Les organisations communautaires et les comités ont été puissamment réactivés pour la circonstance; les maigres capacités financières ont été mobilisées et la totalité de la communauté a participé aux opérations visant à faire face aux conséquences de l'inondation. Nulle part ailleurs le succès de cette mobilisation n'a été plus patent qu'à Deim Mayo, comme l'a montré l'expérience du Conseil des représentants des ethnies du sud. Ce cas est particulièrement intéressant dans la mesure où il permet de rectifier les préjugés traditionnels sur la passivité de la population pauvre (personnes déplacées, travailleurs journaliers, petits paysans, etc.) face aux diverses contraintes qui les oppressent, qu'elles soient le fait de l'environnement naturel ou celui du système socio-économique et politique. Bien plus, le cas du CRTS montre à quel point il importe de prendre les initiatives populaires comme point de départ de tout projet de reconstruction ou de développement.

\section{NOTES}

1. Les données fournies dans cet article sont extraites d'un rapport sur « Les mécanismes d'autoassistance développés par des urbains pauvres de Khartoum face aux inondations de 1988 : le cas de Deim Mayo ", rédigé par l'auteur et par Al-Wathig Kamir en 1989 pour l'ONG OXFAM America (cité en abrégé « Rapport 1989 »). 
INDEX

Mots-clés : Soudan

\section{AUTEUR}

MUSTAFA BABIKER

Université de Khartoum 